\title{
Effects of icariin on the alkline phosphatase activity of human periodontal ligament cells inhibited by lipopolysaccharide
}

\author{
XUE-CHAO LV ${ }^{1}$, LIANG-JIA BI ${ }^{2}$, YING JIANG ${ }^{1}$ and XIANG WANG ${ }^{3}$ \\ ${ }^{1}$ Department of Pediatric Dentistry, School of Stomatology; ${ }^{2}$ Department of Stomatology, \\ The Fourth Affiliated Hospital, Harbin Medical University, Harbin, Heilongjiang 150001; ${ }^{3}$ Department of Tooth Planting, \\ School of Stomatology, The Second Affiliated Hospital, Harbin Medical University, Harbin, Heilongjiang 150086, P.R. China
}

Received June 4, 2013; Accepted August 21, 2013

DOI: $10.3892 / \mathrm{mmr} .2013 .1677$

\begin{abstract}
Icariin (ICA), a flavanoid isolated from herbal Epimedium, has multiple biological activities. The present study investigated the effects of ICA on the proliferation and alkaline phosphatase (ALP) activity (an index for PDLC differentiation) of human periodontal ligament cells (hPDLCs) inhibited by lipopolysaccharide (LPS). hPDLCs were cultured in vitro and stimulated with various concentrations of ICA. The proliferation ability of hPDLCs was detected by an MTT assay. The activity of ALP was determined by the p-Nitrophenyl phosphate method, and the expression of ALP was analyzed by reverse transcription polymerase chain reaction and western blot analysis. ICA exhibited a dose-dependent effect on the proliferation of hPDLCs in a suitable concentration range, from $10^{-6}$ to $10^{-8} \mathrm{~mol} / \mathrm{l}$, and with a mediate optimal concentration $\left(10^{-6} \mathrm{~mol} / \mathrm{l}\right)$. The alkaline phosphatase activity was markedly inhibited in $10 \mu \mathrm{g} / \mathrm{ml}$ LPS-treated PDLCs and this inhibition was suppressed in the presence of icariin at a concentration of $10^{-6} \mathrm{~mol} / \mathrm{L}$ following prolonged treatment (96 h). Therefore, this study provided insight into the use of ICA for periapical tissue regeneration.
\end{abstract}

\section{Introduction}

Periodontitis is one of the most widespread infectious diseases in humans. It is the predominant cause of tooth loss and is associated with a number of systemic diseases, such as

Correspondence to: Liang-Jia Bi, Department of Stomatology, The Fourth Affiliated Hospital, Harbin Medical University, No. 37 Yiyuan Street, Nangang, Harbin, Heilonjiang 150001, P.R. China

E-mail: biliangjia13@126.com

Abbreviations: hPDLCs, human periodontal ligament cells; ALP, alkaline phosphatase; ICA, icariin; LPS, lipopolysaccharide; RT-PCR, reverse transcription polymerase chain reaction

Key words: icariin, periodontal ligament cells, proliferation, alkaline phosphatase, lipopolysaccharide diabetes and cardiovascular disease (1). Periodontitis is stimulated by a variety of factors. For example, lipopolysaccharide (LPS), which acts as an endotoxin and elicits strong immune responses, is important in the pathogenesis of periodontitis. LPS directly induces tumor necrosis factor- $\alpha$ release from macrophages, and is the leading stimulus that initiates the host response in the periodontal pocket and activates macrophages to release proinflammatory cytokines (2-5). Moreover, LPS was observed to exhibit a significant cytotoxic effect on periodontal ligament stem cells (PDLSCs), and affect the self-renewal and osteogeneic differentiation potential of PDLSCs (6).

Alkaline phosphatase (ALP) activity in periodontal ligament cells (PDLCs) is an index for osteogeneic differentiation (7). The activity of ALP isoenzymes were observed to be correlated with cementum formation and root development, and mice lacking ALP demonstrated cementum formation inhibition (8-10). In addition, high ALP activity was observed in the periodontal ligament due to the constant renewal of the tissue or pathological conditions. Furthermore, patients with chronic periodontitis exhibited increased ALP activity in the serum (11), which indicated the possible association between ALP activity in PDLCs and periodontitis. Previous studies have demonstrated that LPS diminishes ALP activity in PDLCs, induces the subtype change and inhibits PDLC differentiation (12). Increased ALP activity appeared to be correlated with subclinical recurrent inflammation and further healing or remodeling of the periodontal tissue (13). Thus, improving ALP activity in periodontal ligaments may be applicable for periodontal tissue regeneration and repair.

Icariin (ICA) is the predominant active ingredient of Herba Epimedii, which is a herb used in traditional Chinese and alternative medicine. ICA increases trabecular bone mineral density in ovariectomized rats and stimulates osteoblastic cell proliferation and differentiation (14). Other studies have demonstrated that ICA and its glycosides accelerated osteoblastic but suppressed osteoclastic differentiation (15). In the present study, the effect of ICA on human PDLCs (hPDLCs) inhibited by LPS was investigated, with the aim of identifying a therapeutic agent for the treatment of periapical disease resulting from bacterial infection. 


\section{Material and methods}

hPDLC isolation and culture. Human tissue samples were collected from the clinically healthy teeth of 11-14 adolescents who had undergone teeth extraction for orthodontic treatment, no history of periodontal disease and a relatively healthy periodontium. The periodontal ligament tissues were obtained as remnants or discarded tissues following routine dental procedures at the Second Affiliated Hospital of. All protocols for the handling of human tissue were approved by the Research Ethics Committee of Harbin Medical University (Harbin, China) and written informed consent was obtained from the families of the patients. The isolation and culture of human mesenchymal stem cells from healthy periodontal ligament tissues was performed as previously described (16). Briefly, tissues were treated aseptically and incubated overnight at $4^{\circ} \mathrm{C}$ with $2 \mathrm{mg} / \mathrm{ml}$ dispase (Sigma-Aldrich, St. Louis, Mo, USA) and $4 \mathrm{mg} / \mathrm{ml}$ collagenase IV (Worthington Biochemical, Lakewood, NJ, USA). The dissociated cell suspension was filtered through a 70- $\mu \mathrm{m}$ cell strainer (Falcon; BD Biosciences, Franklin Lakes, NJ, USA), plated on nontreated $10-\mathrm{cm}$ petri dishes (VWR International, West Sussex, UK) with complete $\alpha$-minimal essential medium (Invitrogen Life Technologies, Carlsbad, CA, USA) containing 20\% fetal bovine serum (Clontech Laboratories Inc., Mountain View, CA, USA), $100 \mathrm{U} / \mathrm{ml}$ penicillin, $100 \mu \mathrm{g} / \mathrm{ml}$ streptomycin (Invitrogen Life Technologies), $2 \mathrm{M}$ L-glutamine, $100 \mathrm{mM}$ nonessential amino acid and $550 \mu \mathrm{M}$ 2-mercaptoethanol (Sigma-Aldrich). The suspension was cultured at $37^{\circ} \mathrm{C}$ in a humidified tissue culture incubator with $5 \% \mathrm{CO}_{2}$ and $95 \% \mathrm{O}_{2}$. After $72 \mathrm{~h}$, the nonadherent cells were removed. The plastic-adherent confluent cells were passaged with $0.05 \%$ trypsin containing $1 \mathrm{mM}$ EDTA and continuously subcultured and maintained in complete growth medium. Cells from the fourth to sixth passages were used in the experiments. Cells from the third passage were fixed by $10 \%$ formaldehyde solution and stained with vimentin and keratin according to standard immunohistochemical methods.

MTT proliferation assay. hPDLCs were seeded in a 96-well plate $\left(2 \times 10^{3}\right.$ cells/well $)$ with varied doses of ICA $\left(0,10^{-5}, 10^{-6}\right.$, $10^{-7}, 10^{-8}$ and $\left.10^{-9} \mathrm{~mol} / \mathrm{l}\right)$. After $96 \mathrm{~h}, 20 \mu 1$ of MTT was added and the cells were incubated for $4 \mathrm{~h}$. Following the addition of $150 \mu 1$ dimethylsulfoxide, the cells were agitated for $10 \mathrm{~min}$, and the concentration was analyzed by measuring the absorbance at $490 \mathrm{~nm}$ with an iMark microplate reader (Bio-Rad, Hercules, CA, USA).

ALP activity assay. An ALP staining kit (Sigma-Aldrich) was used. Subsequent to fixation with $70 \%$ ethanol, cells were incubated with a solution of $0.25 \%$ naphthol AS-BI phosphate and 0.75\% Fast Red Violet LB Base dissolved in 0.1 M Tris buffer (pH 9.3). The ALP activity assay was conducted according to the manufacturer's instructions and normalized on the basis of protein concentrations.

Reverse transcription polymerase chain reaction $(R T-P C R)$. Total RNA was isolated from cultured cells undergoing osteogenic differentiation using an RNeasy Mini kit (Qiagen, Valencia, CA, USA). Adipocyte- and osteocyte-specific genes were amplified using the One-Step
RT-PCR kit (Qiagen). The specific primers used were as follows: Forward, 5'-AGGGCTGTAAGGACATCG-'3 and reverse, 5'-GGAGTGCTTGTATCTCGGTT-3' for ALP; and forward, 5'-CATTGCCGACAGGATGCA-3' and reverse, 5-'CATCTGCTGGAAGGTGGACAG-3' for $\beta$-actin.

Western blot analysis. Cells were lysed with buffer containing $50 \mathrm{mM}$ Tris- $\mathrm{HCl}$ (pH 7.5), $5 \mathrm{mM}$ EDTA, $150 \mathrm{mM} \mathrm{NaCl}, 0.5 \%$ Triton X-100, $10 \mathrm{mM}$ sodium fluoride, $20 \mathrm{mM}$ 2-ME, $250 \mu \mathrm{M}$ sodium orthovanadate, $1 \mathrm{mM}$ phenylmethylsulfonyl fluoride and complete protease inhibitor mixture (Sigma-Aldrich), and were incubated at $4^{\circ} \mathrm{C}$ for $1 \mathrm{~h}$. The lysates were ultrasonicated (AIS92-IIDL ultrasonicator; Aismir, Beijing, China) and centrifuged at $12,000 \mathrm{x}$ g for $10 \mathrm{~min}$. Protein concentrations were determined using the bicinchoninic acid method. Proteins (50-100 $\mu \mathrm{g}$ ) were separated on 8-10\% polyacrylamide-sodium dodecyl sulfate gels and electroblotted onto nitrocellulose membranes (Hybond ECL; Amersham Pharmacia, Picastaway, NJ, USA). Subsequent to blocking with Tris-buffered saline and 5\% nonfat dry milk for $2 \mathrm{~h}$, the membrane was incubated overnight at $4^{\circ} \mathrm{C}$ with antibodies against human ALP (mouse ant-human; Santa Cruz Biotechnology, Inc., Santa Cruz, CA, USA) followed by incubation with a horseradish peroxidase-conjugated secondary antibody (goat anti-mouse; 1:2,000; Pierce, Rockford, IL, USA) for $45 \mathrm{~min}$ at room temperature, and the signals were visualized by enhanced chemiluminescence detection. As a loading control, the blots were reprobed with a specific antibody against human $\beta$-actin (mouse anti-human; dilution, 1:5,000; Santa Cruz Biotechnology, Inc.).

Statistical analysis. Statistical significance was assessed by two-tailed Student's t-test or analysis of variance. $\mathrm{P}<0.05$ and $\mathrm{P}<0.01$ were considered to indicate a statistically significant difference.

\section{Results}

Isolation of hPDLCs. Following primary culture for 4-10 days, fibroblast-like cells with a long fusiform shape emerged beside the tissue block (Fig. 1A). When subcultured to the fourth generation, cells adhered to the bottom of the culture plate and were observed to exhibit a star- or long fusiform-like shape under the microscope. Cell cytoplasm was plump with round or oval nuclei. Cell arrangement was observed to be in a gyrate or radial shape (Fig. 1B). To confirm the origin of cultured cells, various types of stains were applied. Hematoxylin and eosin-stained cell bodies showed long fusiform- or star-like shapes. Nuclei were rounded or oval-shaped and located in the center of the cell body. Cytoplasm stained with anti-vimentin polyclonal antibody appeared brown, while keratin was not stained (Fig. 1D and E). These results suggested that isolated cells had an interstitial opposed to an epithelial origin.

Effects of icariin on hPDLC proliferation. As shown in Table I, icariin accelerated the proliferation of hPDLCs when the concentration was between $10^{-5}$ and $10^{-6} \mathrm{~mol} / 1(\mathrm{P}<0.01$ compared with the control group). The effect appeared to be concentration-dependent within this concentration range. Lower icariin concentrations exhibited no significant effect on 

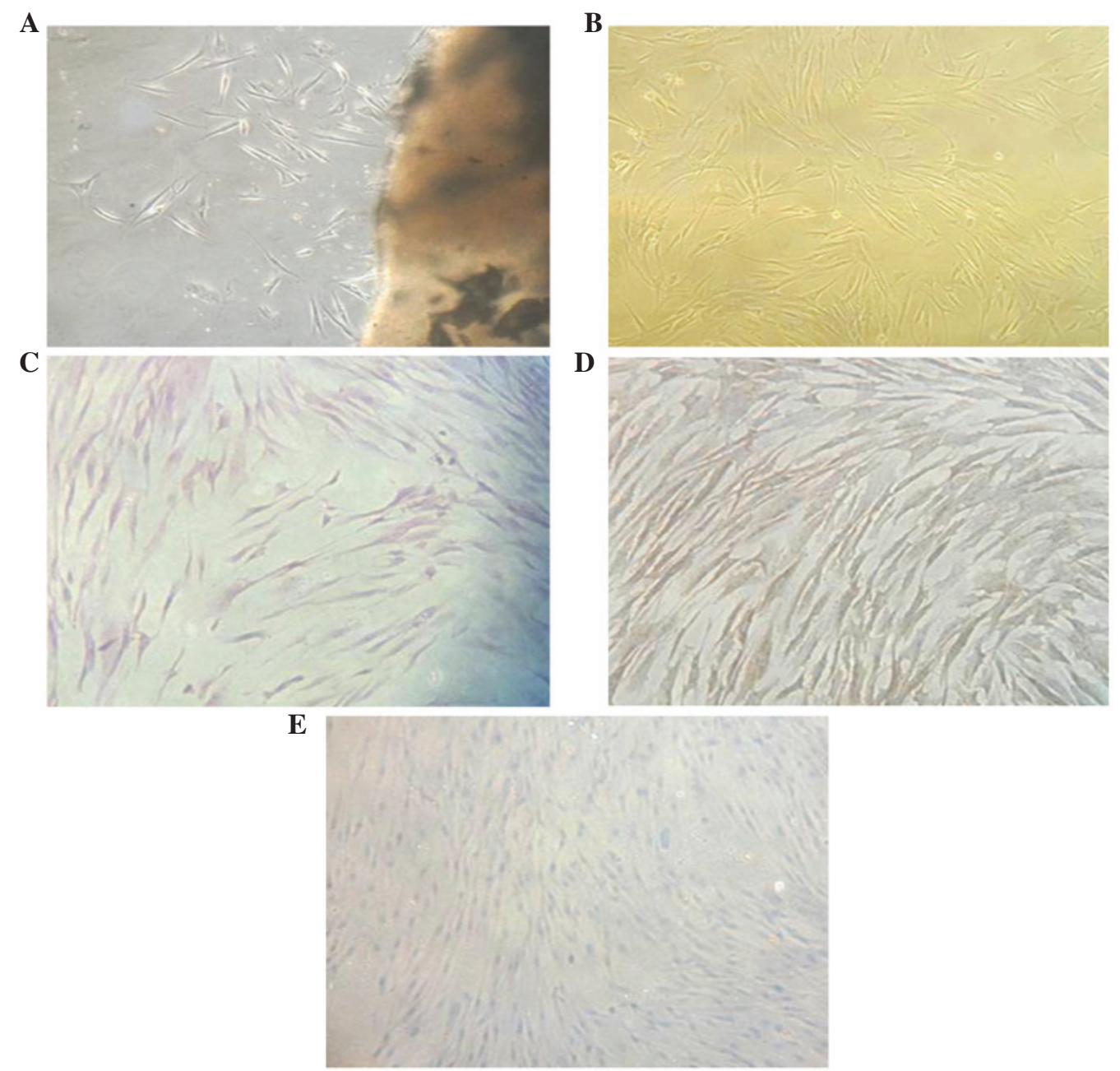

Figure 1. Isolation and identification of hPDLCs. (A) Primary cultured cells. (B) Fourth-passage subcultured cells. (C) Fourth-passage cells stained by hematoxylin and eosin. (D) Third-passage cells stained by vimentin antibodies. (E) Third-passage cells stained by keratin antibodies but appeared marginally colored. hPDLCs, human periodontal ligament cells. (A and B) Magnification, x100; (C-E) magnification, x400.

Table I. Effect of ICA concentration on hPDLC proliferation.

\begin{tabular}{lc}
\hline ICA concentration $(\mathrm{mol} / \mathrm{l})$ & Proliferation ability (OD490) \\
\hline 0 & $0.36 \pm 0.04$ \\
$10^{-5}$ & $0.32 \pm 0.04^{\mathrm{a}}$ \\
$10^{-6}$ & $0.49 \pm 0.04^{\mathrm{b}}$ \\
$10^{-7}$ & $0.40 \pm 0.05^{\mathrm{a}}$ \\
$10^{-8}$ & $0.36 \pm 0.02$ \\
$10^{-9}$ & $0.36 \pm 0.03$
\end{tabular}

${ }^{\mathrm{a}} \mathrm{P}<0.05$ and ${ }^{\mathrm{b}} \mathrm{P}<0.01$ compared with $0 \mathrm{~mol} / \mathrm{l}$ group. Data are presented as the mean $\pm \mathrm{SD}(\mathrm{n}=8)$. ICA, icariin; hPDLC, human periodontal ligament cell.

hPDLC proliferation while higher concentrations inhibited the division of hPDLCs $(\mathrm{P}<0.05)$.

Icariin promotes differentiation of hPDLCs inhibited by $L P S$. High ALP activity is a well-known index for hPDLC ossification. Previous studies have shown that LPS inhibited
Table II. ALP activity of different groups after $96 \mathrm{~h}$ incubation..

\begin{tabular}{lc}
\hline Groups & $\begin{array}{c}\text { ALP activity } \\
(\mathrm{U} / \mu \mathrm{l} / \mu \mathrm{g} \text { protein })\end{array}$ \\
\hline $\mathrm{N}$ & $3.55 \pm 0.41$ \\
$\mathrm{LPS}$ & $1.18 \pm 0.38$ \\
$\mathrm{ICA}+\mathrm{LPS}$ & $2.1 \pm 0.44$ \\
\hline
\end{tabular}

Data are presented as the mean \pm SD $(n=6)$. ALP, alkaline phosphatase. N, control group; LPS, lipopolysaccharide-treated group; ICA+LPS, icariin- and LPS-treated group.

hPDLC differentiation and this process was associated with ALP activity decreases. When icariin $\left(10^{-5} \mathrm{~mol} / \mathrm{l}\right)$ was added to media containing LPS, the ALP activity was not changed at $24 \mathrm{~h}$; however, following incubation for $96 \mathrm{~h}$, hPDLCs exhibited high ALP activity (Table II).

To identify at which level ALP activity returned to normal levels, RT-PCR and western blot analysis were used to measure the gene expression and protein levels 
A

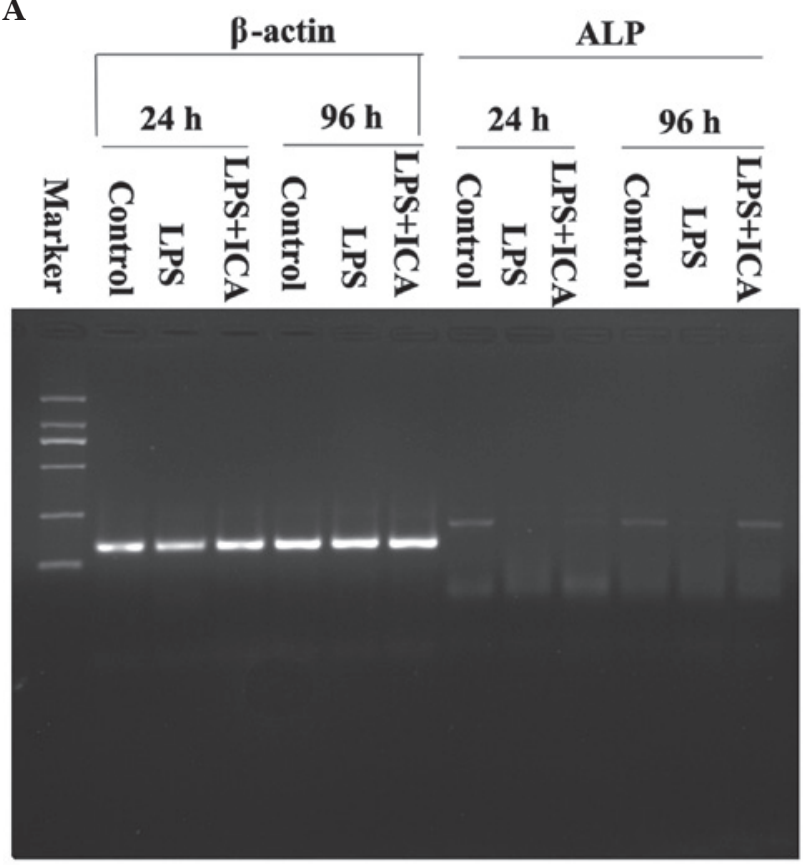

B

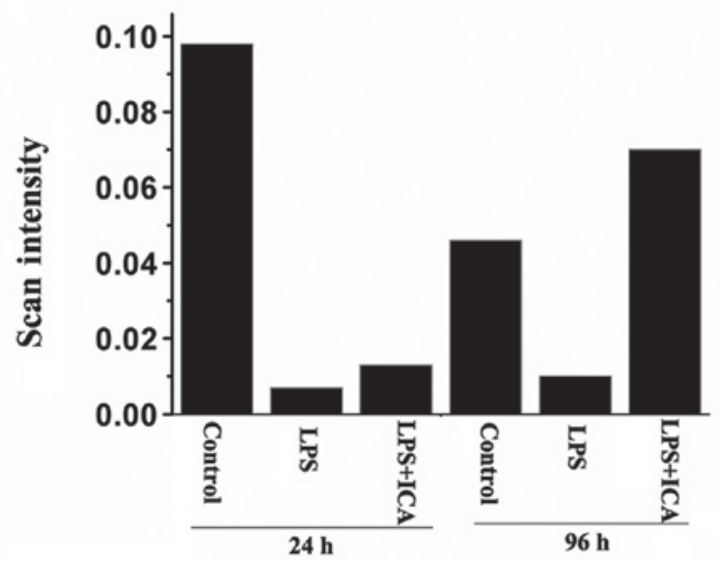

Figure 2. Reverse transcription polymerase chain reaction (RT-PCR) detection of alkaline phosphatase (ALP) gene expression. (A) Periodontal ligament cells (PDLCs) were treated with lipopolysaccharide (LPS) or LPS and icariin (ICA). Cells were harvested following treatment for 24 or $96 \mathrm{~h}$, RNA was isolated and RT-PCR was conducted using $\beta$-actin as a reference. (B) DNA agarose gels were run and band intensities were scanned. The experiment was replicated. Actin served as the internal control.

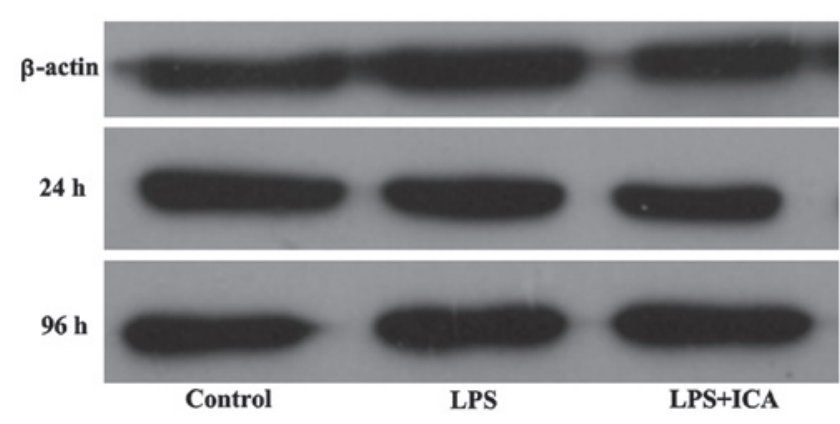

Figure 3. Western blot analysis of alkaline phosphatase (ALP) expression Cells were harvested and total proteins were extracted. Equal quantities of protein were loaded and electrophoresis was carried out. ALP protein levels were analyzed following treatment for 24 and $96 \mathrm{~h}$. LPS, lipopolysaccharide-treated group; LPS+ICA, LPS and icariin co-treatment group. of ALP, respectively. The RT-PCR results showed that no significant $A L P$ expression difference was detected after $24 \mathrm{~h}$. Differentiation emerged between the LPS group and icarrin-treated group after $96 \mathrm{~h}$. The ALP activity of the icariin group was greater than that observed in the control group $(\mathrm{P}<0.01)$ (Fig. 2).

These results suggested that the $A L P$ gene expression level that had been inhibited by LPS was recovered by icariin following prolonged exposure. These results were similar in the western blot analysis (Fig. 3). LPS suppressed ALP expression at 24 and $96 \mathrm{~h}$, while icariin accelerated this inhibition at $24 \mathrm{~h}$, but markedly increased the expression at $96 \mathrm{~h}$, exceeding the level of the control group $(\mathrm{P}<0.01)$. Thus, the ALP activity fluctuation was directly correlated with the protein level of the enzyme, which was controlled by the ALP expression.

\section{Discussion}

The apex dentis lesion of the un-develped permanent teeth of teenagers could be medically induced to apical foramen occlusion. Yang et al (17) observed that the occlusion was hindered by the accumulation of dentin, osteoid dentin, cementum and mineralization, which differentiated from parodontium connective tissue. Parodontium predominantly consists of PDLCs and collagenous fiber (18). PDLCs are heterogenic multipotential stem cells that possess important biological functions. PDLCs proliferate and differentiate into osteoblasts, which synthesize periodontal ligament, alveolar bone and cementum, thus repairing and regenerating periapical tissue (19).

Herba epimedii is a traditional Chinese herb that has multiple medical effects. Modern pharmacological studies have suggested that icariin extracted from herba epimedii inhibited osteoclast function and accelerated the proliferation and differentiation of osteoblasts (20). Icariin also decreased the cytotoxicity resulting from LPS; however, the detailed mechanism of this action remains to be elucidated. In the present study, the effects of icariin on PDLC proliferation in an in vitro culture were investigated. The results suggested that icariin significantly accelerated PDLC proliferation at a concentration range between $10^{-7}$ and $10^{-6} \mathrm{~mol} / 1$ in a concentration-dependent manner. A concentration of $10^{-6} \mathrm{~mol} / \mathrm{l}$ exhibited the strongest effect.

ALP activity is one of the most important markers for ossification (7). ALP conducts dephosphorylation, destroys inhibitors of mineralization and acts as a calcium binding protein or phosphate transporter to promote ossification. ALP activity in PDLCs indicates ossification transformation. LPS, a pathogenic sugar residue isolated specifically on Gram negative bacteria, is a toxic antigen to cells in the periapical tissue (21). LPS is important in the development of periapical diseases (7). In hPDLCs, LPS was observed to decrease the ALP activity, leading to a loss of potential to differentiate into osteoblasts and cementoblasts. Thus, increasing the ALP activity in inhibited PDLCs is crucial for PDLC regeneration and differentiation. The addition of icariin to LPS-inhibited PDLCs increased ALP activity following exposure for $>96 \mathrm{~h}$, while a short exposure period $(\sim 24 \mathrm{~h})$ did not show a marked increase. The molecular mechanism underlying the 
antagonism was suggested to be mediated by icariin increasing ALP gene and protein expression levels, which promotes ALP activity. Improved ALP activity induced PDLC renewal and the potential for differentiation. This provided insight into the use of icariin as a treatment for periapical disease resulting from LPS.

\section{Acknowledgements}

This study was supported by the Natural Science Foundation of Heilongjiang province (grant no.41400649-6-12281).

\section{References}

1. Kinane DF and Marshall GJ: Periodontal manifestations of systemic disease. Aust Dent J 46: 2-12, 2001.

2. Roberts FA, Hockett RD Jr, Bucy RP, et al: Quantitative assessment of inflammatory cytokine gene expression in chronic adult periodontitis. Oral Microbiol Immunol 12 336-344, 1997.

3. Gamonal J, Acevedo A, Bascones A, et al: Levels of interleukin-1 beta, -8 , and -10 and RANTES in gingival crevicular fluid and cell populations in adult periodontitis patients and the effect of periodontal treatment. J Periodontol 71: 1535-1545, 2000.

4. Thammasitboon K, Goldring SR and Boch JA: Role of macrophages in LPS-induced osteoblast and PDL cell apoptosis. Bone 38: 845-852, 2006.

5. Kumada H, Haishima Y, Umemoto T, et al: Structural study on the free lipid A isolated from lipopolysaccharide of Porphyromonas gingivalis. J Bacteriol 177: 2098-2106, 1995.

6. Cho JH, Lee SK, Lee JW and Kim EC: The role of heme oxygenase-1 in mechanical stress-and lipopolysacharide-induced osteogenic differentiation in human periodontal ligament cells. Angle Orthod 80: 552-559, 2010.

7. Murakami Y, Kojima T, Nagasawa T, et al: Novel isolation of alkaline phosphatase-positive subpopulation from periodontal ligament fibroblasts. J Periodontol 74: 780-786, 2003.

8. Groeneveld MC, Everts V and Beertsen W: Alkaline phosphatase activity in the periodontal ligament and gingiva of the rat molar: its relation to cementum formation. J Dent Res 74: 1374-1381, 1995.
9. Beersten W, vandenBos T and Everts V: Root development in mice lacking functional tissue non-specific alkaline phosphatase gene: inhibition of acellullar cementum formation. J Dent Res 78 1221-1229, 1999.

10. Anan H, Akamine A, Maeda K: An enzyme histochemical study of the behavior of rat bone cells during experimental apical periodontitis. J Endod 19: 83-86, 1993.

11. Gibert P, Tramini P, Sieso V and Piva MT: Alkaline phosphatase isoenzyme activity in serum from patients with chronic periodontitis. J Periodontal Res 38: 362-365, 2003.

12. Zhang Feng-Qiu, Wu Zhi-Fen, Wan Ling, et al: Effect of lipopolysaccharides on proliferation and alkaline phosphatase activity of periodontal ligament cells. Chinese Journal of Conservative Dentistry 13: 27-19, 2003 (In Chinese).

13. Perinetti G, Paolantonio M, Femminella B, Serra E and Spoto G: Gingival crevicular fluid alkaline phosphatase activity reflects periodontal healing/recurrent inflammation phases in chronic periodontitis patients. J Periodontol 79: 1200-1207, 2008.

14. Mok SK, Chen WF, Lai WP, et al: Icariin protects against bone loss induced by oestrogen deficiency and activates oestrogen receptor-dependent osteoblastic functions in UMR 106 cells. Br J Pharmacol 159: 939-949, 2010.

15. Huang J, Yuan L, Wang X, Zhang TL and Wang K: Icariin and its glycosides enhance osteoblastic, but suppress osteoclastic, differentiation and activity in vitro. Life Sci 81: 832-840, 2007.

16. Liu D, Xu J, Liu O, Fan Z, et al: Mesenchymal stem cells derived from inflamed periodontal ligaments exhibit impaired immunomodulation. J Clin Periodontol 39: 1174-1182, 2012.

17. Yang SF, Yang ZP and Chang KW: Continuing root formation following apexifiction treatment. Endod Dent Traumatol 6 : 232-235, 1990.

18. Lekic P and McCulloch CA: Periodontal ligament cell populations: the central role of fibroblasts in creating a unique tissue. Anat Rec 245: 327-341, 1996.

19. Chantarawaratit P, Sangvanich P, Banlunara W, Soontornvipart K and Thunyakitpisal P: Acemannan sponges stimulate alveolar bone, cementum and periodontal ligament reneberation in a canine class II furcation defect model. J Periodontal Res 48: 224-232, 2013

20. Hseih TP, Sheu SY, Sun JS and Chen MH: Icariin inhibits osteoclast differentiation and bone resorption by suppression of MAPKs/NF- $\kappa$ B regulated HIF- $1 \alpha$ and PGE(2) synthesis. Phytomedicine 18: 176-185, 2011.

21. Hong CY, Lin SK, Kok SH, et al: The role of lipopolysaccharide in infectious bone resorption of periapical lesion. J Oral Pathol Med 33: 162-169, 2004. 